\section{SAT0315 ABATACEPT IN PSORIATIC ARTHRITIS: A SINGLE CENTRE, PLACEBO-CONTROLLED, CROSSOVER STUDY IN 20 PATIENTS; A PILOT PROTEOMIC FEASIBILITY STUDY}

A. Kwasnik ${ }^{1}$, S. Pennington ${ }^{1}$, O. FitzGerald ${ }^{2} .{ }^{1}$ Conway Institute of Biomolecular and Biomedical Research, University College Dublin; ${ }^{2}$ Department of Rheumatology, St. Vincent's University Hospital, Dublin, Ireland

Background: Psoriatic arthritis (PsA) is a multifaceted inflammatory disease that affects approximately $0.25 \%$ of the global population ${ }^{1}$. A range of disease modifying drugs has been used to treat PsA including Abatacept. Unfortunately, not al PsA patients respond to Abatacept treatment and some patients may be intolerant, thus there is an urgent need for the improved selection of patients who are likely to respond Abatacept treatment.

Objectives: This pilot project seeks to demonstrate the potential with which MS based proteomics strategies might be used to discover proteins that may discriminate responders from non-responders to treatment with Abatacept. Using synovia samples collected at baseline and a label-free nLC-MS/MS strategy, candidate proteins were identified. These proteins together with other proteins identified in previous studies were further evaluated by developing multiplexed MRM assays to quantify the proteins both before and during Abatacept treatment (at 2 and 6 months).

Methods: Baseline samples from 6 patients were prepared according to the FASP protocol and analysed by Q-Exacive. MS data was analysed using MaxQuant (v. 1.4.1.2.) and Perseus (v. 1.5.0.8). For the evaluation of candidate proteins Skyline (v. 3.7.0.11317) was used to develop multiplexed MRM assays for these newly identified proteins and to analyse the MRM data acquired on high sensitivity triple quadrupole mass spectrometer (Agilent 6495)

Results: 41 proteins were shown to be differentially expressed at baseline between responders $(n=5)$ and the non-responder $(n=1)$. Of these proteins, 15 were elevated and 26 were reduced in the non-responder. A sub-set of the candidate proteins identified here $(n=41)$ and some proteins identified in our previous PsA related studies ${ }^{2}$ was used to develop an MRM assay targeting total 114 proteins. The MRM assay was developed using our stringent MRM assay standards. Six candidate proteins, ANXA1, ANXA2, S100A10, LMNA, CADH5 and MYL6 have been shown to be differentially expressed in synovial samples collected at 2 months when compared to 6 months. A further 5 proteins, ANXA5, RS18, TAGL, TRFL and HPT were differentially expressed between responders and non-responder at the 2 month time point but most importantly, these five proteins have shown a similar pattern of expression following four months of Abatacept treatment.

Conclusions: Although the small number of patient samples in this pilot study limits the biological significance of these findings, the data highlights some of the significant advantages of unbiased LC-MS/MS protein discovery and of multiplexed MRM assays. These advantages include reproducible and robust coverage of a large number of proteins in small synovial tissue samples and a workflow that supports rapid development of optimised multiplexed assays targeted to proteins of interest.

\section{REFERENCES:}

[1] McArdle A, Pennington S, FitzGerald O. Clinical Features of Psoriatic Arthritis: a Comprehensive Review of Unmet Clinical Needs. Clin. Rev. Allergy Immunol 2017;1-24.

2] Ademowo OS. et al. Discovery and confirmation of a protein biomarker panel with potential to predict response to biological therapy in psoriatic arthritis. Ann. Rheum. Dis 2016 75;234-41.

Disclosure of Interest: A. Kwasnik: None declared, S. Pennington: None declared, O. FitzGerald Grant/research support from: Study supported by BMS DOI: 10.1136/annrheumdis-2018-eular.4489

\section{SAT0316 RAPID AND SUSTAINED IMPROVEMENTS IN BOTH SKIN AND MUSCULOSKELETAL SYMPTOMS CORRELATES WITH IMPROVED QUALITY OF LIFE IN PATIENTS WITH ACTIVE PSORIATIC ARTHRITIS}

A.F. Kavanaugh ${ }^{1}$, J. Birt' ${ }^{2}$ C.-Y. Lin ${ }^{2}$, O. Benichou ${ }^{3}$, M. Hufford ${ }^{2}$, A.B. Gottlieb ${ }^{4}$ ${ }^{1}$ Univ of CA San Diego Health System, La Jolla; ${ }^{2}$ Eli Lilly and Company, Indianapolis, USA; ${ }^{3}$ Eli Lilly and Company, Paris, France; ${ }^{4}$ New York Medical College, New York, USA

Background: Psoriatic arthritis (PsA) is a chronic, systemic, autoimmune, inflammatory arthritis condition commonly associated with psoriasis. Several biologic therapies exist for the treatment of PsA, with varying degrees of efficacy and onset of action.

Objectives: The objective of this analysis was to examine whether there is additional value to a patient's overall quality of life if efficacy is achieved early during treatment and sustained.

Methods: Data were analysed from an integrated database of 2 double-blind, phase III SPIRIT trials investigating the efficacy and safety of ixekizumab (IXE), a high-affinity monoclonal antibody selectively targeting interleukin-17A, for patients with active PsA. The integrated database consisted of patients who were biologicnaïve (SPIRIT-P1, NCT01695239) or who had an inadequate response or were intolerant to tumour necrosis factor inhibitors (SPIRIT-P2; NCT02349295). Patients were randomised to placebo $(n=224)$ or 80 mg IXE every $4(n=229)$ or 2 weeks $(n=226)$ after a $160 \mathrm{mg}$ starting dose. Patients included in this post-hoc analysis had baseline $\geq 3 \%$ body surface area (BSA) and at least one visit with a Psoriasis Area and Severity Index (PASI) 75 and American College of Rheumatology (ACR) 20 response; all treatment groups were combined. During the first 24 weeks of the trial, health-related quality of life (HRQoL) was measured by Short Form-36 Health Survey (SF-36) domain scores, the EuroQoL 5 Dimensions Visual Analogue Scale (EQ-5D VAS), and the Work Productivity and Activity Impairment-Specific Health Problem (WPAI-SHP) activity impairment domain. Change in $\mathrm{HRQ}$ oL at Week 24 was modelled using a response surface model with duration of consecutive PASI and ACR responses and their interaction as independent variables. Missing data were imputed using last observation carried forward and non-responder imputation for continuous and categorical endpoints, respectively. Results: Of the 679 placebo- and IXE-treated patients in the SPIRIT trials, 215 $(31.7 \%)$ had baseline $\geq 3 \%$ BSA and $\geq 1$ PASI75 and ACR20 response. Longer consecutive ACR20 responses correlated positively with greater HRQoL improvements, as measured by the EQ-5D VAS (figure 1). Patients with a longer consecutive number of both ACR20 and PASI75 responses had the highest improvements in EQ-5D VAS. This was consistent with 7 of 8 SF-36 domains, as well as the WPAI-SHP activity impairment domain (data not shown).

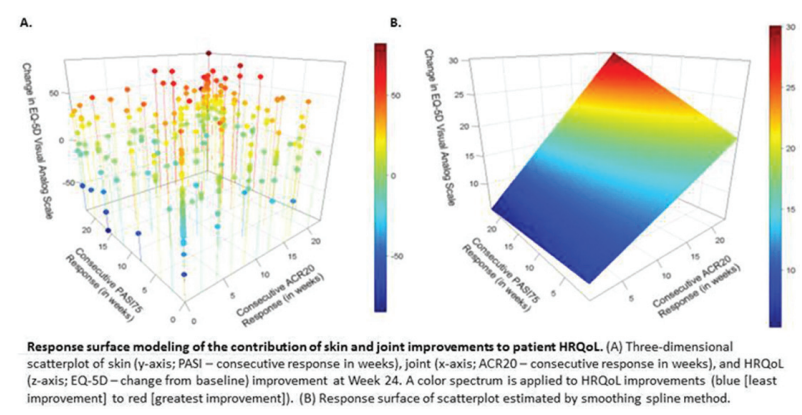

Abstract SAT0316 - Figure 1

Response surface modelling of the contribution of skin and joint improvements to patient HRQoL. (A) Three-dimensional scatterplot of skin (y-axis; PAS - consecutive response in weeks), joint (x-axis; ACR20 - consecutive response in weeks), and HRQoL ( $z$-axis; EQ-5D - change from baseline) improvement at Week 24. A colour spectrum is applied to HRQoL improvements (blue [least improvement] to red [greatest improvement]). (B) Response surface of scatterplot estimated by smoothing spline method.

Conclusions: Early and sustained improvements in the symptoms of PSA correlated positively with improved HRQoL. The greatest HRQoL improvements were achieved when both skin and musculoskeletal symptoms improved early and were sustained.

Disclosure of Interest: A. Kavanaugh Consultant for: Eli Lilly and Company, J. Birt Shareholder of: Eli Lilly and Company, Employee of: Eli Lilly and Company, C.-Y. Lin Shareholder of: Eli Lilly and Company, Employee of: Eli Lilly and Company, O. Benichou Employee of: Eli Lilly and Company, M. Hufford Shareholde of: Eli Lilly and Company, Employee of: Eli Lilly and Company, A. B. Gottlieb Grant/research support from: Janssen, Incyte, Consultant for: Janssen Inc.; Celgene Corp., Bristol Myers Squibb Co., Beiersdorf, Inc., Abbvie, UCB, Novartis, Incyte, Lilly, Reddy Labs, Valeant, Dermira, Allergan, Sun Pharmaceutical Industries

DOI: 10.1136/annrheumdis-2018-eular.2331 


SAT0317

TOFACITINIB IN PATIENTS WITH PSORIATIC ARTHRITIS AND METABOLIC SYNDROME: A POST-HOC ANALYSIS OF PHASE 3 STUDIES

C. Ritchlin ${ }^{1}$, J. Giles ${ }^{2}$, A. Ogdie ${ }^{3}$, J. Gomez-Reino ${ }^{4}$, L. Stockert ${ }^{5}$, P. Young ${ }^{5}$, C. Wang ${ }^{6}$, A.B. Romero ${ }^{7}$, E. Kudlacz ${ }^{6} .{ }^{1}$ University of Rochester Medical Center, Rochester, NY; ${ }^{2}$ Columbia University, New York, NY; ${ }^{3}$ Perelman School of Medicine at the University of Pennsylvania, Philadelphia, PA, USA; ${ }^{4}$ Hospital Clínico Universitario, Santiago de Compostela, Spain; ${ }^{5}$ Pfizer Inc, Collegeville, PA; ${ }^{6}$ Pfizer Inc, Groton, CT, USA; ${ }^{7}$ Pfizer Inc, Barcelona, Spain

Background: Tofacitinib is an oral Janus kinase inhibitor for the treatment of psoriatic arthritis (PsA). PsA is often associated with comorbid metabolic syndrome (MetS), which is linked to increased inflammation and severity of underlying PsA, and higher cardiovascular risk. ${ }^{1,2}$ Patients (pts) with PsA and comorbid MetS frequently demonstrate decreased therapeutic responses and lower probability of achieving minimal disease activity.

Objectives: To compare key efficacy and safety endpoints in tofacitinib-treated pts with PsA and MetS in Phase (P) 3 studies.

Methods: Two double-blind P3 studies enrolled pts with active PsA who either had an inadequate response (IR) to $\geq 1$ conventional synthetic (cs)DMARD and were TNFi-naïve (OPAL Broaden; $n=422 ; 12$ months; NCT01877668) or IR to $\geq 1$ TNFi (OPAL Beyond; $\mathrm{n}=395 ; 6$ months; NCT01882439). Pts were randomised to receive tofacitinib $5 \mathrm{mg} \mathrm{BID}$, tofacitinib $10 \mathrm{mg} \mathrm{BID}$, adalimumab $40 \mathrm{mg}$ Q2W (OPAL Broaden only) or placebo (PBO); all pts continued on a single, stable CsDMARD. In this analysis, data for tofacitinib- and PBO-treated pts were pooled from both studies; efficacy and safety endpoints at Month (M) 3 were descriptively reported according to the presence or absence of MetS at baseline (defined as $>3$ of the following: hypertension, elevated triglycerides, reduced HDL cholesterol, large waist size and elevated fasting glucose levels ${ }^{5}$ ). Efficacy endpoints included: ACR20 response; change from baseline in HAQ-DI; PASI75 response; and changes from baseline in Pt's Global Assessment of Arthritis and C-reactive protein (CRP). Safety endpoints included: treatment-emergent adverse events (AEs); fasting lipid levels (LDL, HDL and total cholesterol, and triglycerides).

Results: This analysis included 294 pts with MetS (tofacitinib $5 \mathrm{mg}, \mathrm{n}=99 ; 10 \mathrm{mg}$, $n=101 ;$ PBO, $n=94$ ) and 416 pts without (tofacitinib $5 \mathrm{mg}, \mathrm{n}=139 ; 10 \mathrm{mg}, \mathrm{n}=135$; PBO, $n=142$ ). At baseline, pts with MetS had a higher mean age (53.2 vs 46.2 years) and mean BMI (33.2 vs $\left.27.3 \mathrm{~kg} / \mathrm{m}^{2}\right)$, and a greater proportion of pts with MetS had a CRP level $>2.87 \mathrm{mg} / \mathrm{L}$ (upper limit of normal) $(67.7 \mathrm{vs} 58.9 \%$ ) and were taking lipid-lowering medications (Day $1: 26.5$ vs $3.8 \%$ ). Tofacitinib efficacy was generally similar in pts with and without MetS (Table). LDL, HDL and total cholesterol, and triglyceride levels generally increased from baseline to M3 (Table). Among pts with MetS, AEs occurred in $55.6 \%$ treated with tofacitinib $5 \mathrm{mg}$ (serious AEs [SAEs], $2 \%$ ) and in $42.6 \%$ treated with tofacitinib $10 \mathrm{mg}$ (SAEs, $2 \%$ ). Among those without MetS, AEs occurred in $42.4 \%$ treated with tofacitinib $5 \mathrm{mg}$ (SAEs, $1.4 \%$ ) and in $54.8 \%$ treated with tofacitinib $10 \mathrm{mg}$ (SAEs, $1.5 \%)$.

Abstract SAT0317 - Table 1. Efficacy and safety endpoints at Month 3 in OPAL Broaden and OPAL Beyond

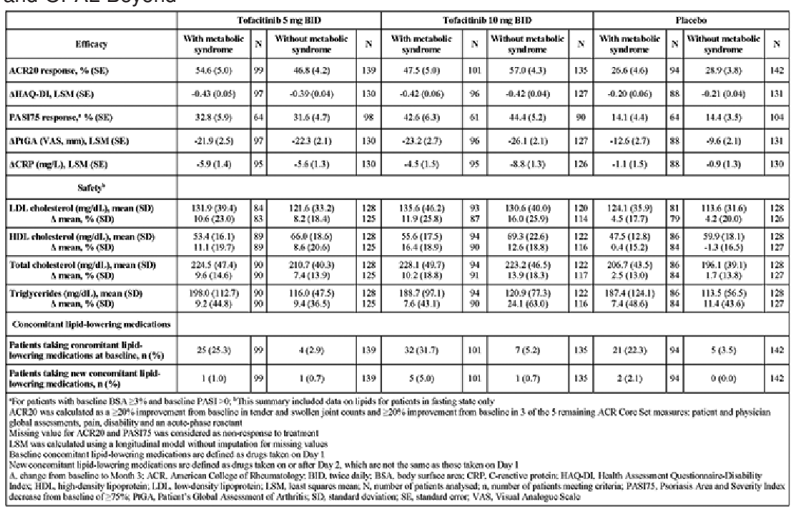

Conclusions:

Across 2 P3 studies, tofacitinib showed generally similar efficacy and safety in pts with PsA with or without MetS.

\section{REFERENCES:}

[1] Haroon M, et al. J Rheumatol 2014; 41:1357-65.

[2] Haroon M, et al. J Rheumatol 2016;43:463-4.

[3] Stober C, et al. Rheumatology 2018;57:158-63.

[4] Costa L, et al. Immunol Res 2015;61(1-2):147-53.

[5] Alberti KG, et al. Circulation 2009;120:1640-5.

Acknowledgements: Study sponsored by Pfizer Inc. Medical writing support was provided by S Piggott of CMC and funded by Pfizer Inc.

Disclosure of Interest: C. Ritchlin Grant/research support from: AbbVie, Amgen and UCB, Consultant for: AbbVie, Amgen, Celgene, Janssen, Novartis, Pfizer Inc, Sun and UCB, J. Giles: None declared, A. Ogdie Grant/research support from: Novartis, Consultant for: Novartis, Pfizer Inc and Takeda, J. Gomez-Reino Grant research support from: AbbVie, MSD, Pfizer Inc and Roche, Consultant for: Pfizer Inc, Speakers bureau: AbbVie, Biogen, Bristol-Myers Squibb, Janssen, MSD, Pfizer Inc and Roche, L. Stockert Shareholder of: Pfizer Inc, Employee of: Pfizer Inc, P. Young Shareholder of: Pfizer Inc, Employee of: Pfizer Inc, C. Wang Shareholder of: Pfizer Inc, Employee of: Pfizer Inc, A. B. Romero Shareholder of: Pfizer Inc, Employee of: Pfizer Inc, E. Kudlacz Shareholder of: Pfizer Inc, Employee of: Pfizer Inc

DOI: 10.1136/annrheumdis-2018-eular.3242

\section{SAT0318 DO PATIENTS IN REMISSION IN PSORIATIC ARTHRITIS, HAVE LESS FATIGUE? AND DOES THIS DEPEND ON THE DEFINITION OF REMISSION? AN ANALYSIS OF 304 PATIENTS}

C. Gorlier, D. Puyraimond-Zemmour, U. Kiltz, A.-M. Orbai, Y.Y. Leung,

P. Palominos, J.D. Cañete, R. Scrivo, A. Balanescu, E. Dernis, S. Talli,

A. Ruyssen-Witrand, M. Soubrier, S. Aydin, L. Eder, I. Gaydukova, E. Lubrano,

L. Coates, U. Kalyoncu, J. Smolen, M. De Wit, L. Gossec. ReFlaP study working group, PARIS, France

Background: Fatigue is a critical element of life impact for patients with Psoriatic Arthritis (PsA) and is not considered in remission definitions. In PsA, remission can be defined using composite scores (Minimal Disease Activity (MDA), Disease Activity in PSoriatic Arthritis (DAPSA) $\leq 4)$, Patient Acceptable Symptom State (PASS), Patient Global Assessment (PGA)(for example $\leq 1 / 10$ ), or as a single for remission item.

Objectives: To explore the relationship between fatigue and remission in PsA, when using different definitions of remission.

Methods: ReFlaP (NCT03119805) is a cross-sectional study in 14 countries of consecutive adult patients with definite PsA and more than 2 years of disease duration. Patient-perceived fatigue was assessed by a 11-point numerical rating scale. Remission status was defined from the physician's perspective as MDA, $\mathrm{DAPSA} \leq 4$ and physician-perceived remission (single question yes/no), and from the patient's perspective as PASS, PGA $\leq 1$ and patient-perceived remission (single question yes/no). We calculated fatigue group means and deltas by remission status and compared these by Student's t test. For known groups validity of each remission definition we used ROC curves and corresponding areas under the curve (AUC).

Results: Of 366 patients, 304 had both fatigue and remission data available: 148 $(49.8 \%)$ were male, mean age was $53.9 \pm 12.3$ years, mean disease duration was $10.8 \pm 7.7$ years; $90.3 \%$ had predominant peripheral disease, $56.3 \%$ were taking methotrexate, $66.5 \%$ a biologic and $19.4 \%$ oral glucocorticoids. Disease activity was moderate: $41.1 \%$ had no current psoriasis skin lesions, mean Tender Joint Count (TJC) was 4.3 \pm 8.9 , mean Swollen Joint Count (SJC) was 2.66 \pm 8.3 , mean Physician's global assessment was $3.0 \pm 2.4$, mean PGA was $4.19 \% \pm 2.7$. 80.6\% patients had DAPSA levels $<28$ (ie, remission, low or moderate disease activity). Mean patient's assessment of fatigue was $4.26 \pm 3.0$. The frequency of remission varied from $17.4 \%$ to $64.8 \%$ (the most stringent definition being DAPSA and the least PASS). Fatigue levels were much lower in remission than non-remission with group differences in fatigue ranging from 1.66 \pm 0.3 ) (Physician remission single question yes/no) to $3.81 \pm 0.3$ (DAPSA remission) (all p<0.0001) (figure 1). Corresponding AUCs ranged from 0.66 (Physician's remission question) to 0.87 (DAPSA). 